\title{
MicroRNA-195 inhibits colorectal cancer cell proliferation, colony-formation and invasion through targeting CARMA3
}

\author{
LING WANG $^{1}$, LIYUAN QIAN ${ }^{1}$, XIAORONG LI $^{1}$ and JIN YAN ${ }^{2}$ \\ ${ }^{1}$ The Second Department of General Surgery; ${ }^{2}$ Department of Nursing, \\ The Third Xiangya Hospital of Central South University, Changsha, Hunan 410013, P.R. China
}

Received September 24, 2013; Accepted April 7, 2014

DOI: $10.3892 / \mathrm{mmr} .2014 .2178$

\begin{abstract}
MicroRNA (miR)-195 has been reported to be a tumor suppressor. Downregulation of miR-195 has been shown to correlate with lymph node metastasis and poor prognosis in colorectal cancer. However, the specific regulatory role of miR-195 in colorectal cancer cells is yet to be elucidated. In the present study, miR-195 expression was significantly reduced in colorectal cancer tissues. Furthermore, CARMA3 was identified as a novel target of miR-195, which was observed to be upregulated in colorectal cancer. In addition, downregulation of miR-195 increased CARMA3 protein expression, whereas miR-195 upregulation suppressed CARMA3 protein expression in SW480 and HT29 colorectal cancer cells. Moreover, overexpression of miR-195 downregulated cell proliferation, colony-formation and invasion in SW480 and HT29 cells, which was reversed upon CARMA3 overexpression. In conclusion, the findings of the present study suggest that miR-195 has a suppressive role in colorectal cancer cells through directly targeting CARMA3. Therefore, CARMA3 may be a potential target for the treatment of colorectal cancer.
\end{abstract}

\section{Introduction}

Colorectal cancer, one of the most common types of cancer in males and females, is a serious demographic and economic problem worldwide (1). The development and progression of colorectal cancer are regulated by a complex cascade of molecular events, which are involved in cell proliferation and invasion (2). Therefore, it is of great importance to explore the molecular mechanisms underlying colorectal carcinogenesis.

MicroRNAs (miRNAs) are a type of endogenous non-coding RNA, which act as endogenous suppressors of translation by directly binding to the 3 ' untranslated region

Correspondence to: Professor Jin Yan, Department of Nursing, The Third Xiangya Hospital of Central South University, 138 Tongzipo Road, Changsha, Hunan 410013, P.R. China E-mail: csuyanjin@163.com

Key words: colorectal cancer, microRNA-195, CARMA3, proliferation, colony-fomation, invasion
(UTR) of target mRNAs (3). Recently, numerous miRNAs have been shown to be involved in the development and progression of colorectal cancer (4). For example, miR-133a has been observed to suppress colorectal cancer through activating the p53/p21 signaling pathway (5), and the inhibition of miR-21 has been found to enhance the susceptibility of chemoresistant colon cancer cells to therapeutic treatment (6). Therefore, miRNAs may be promising candidates or targets for colorectal cancer therapy.

miR-195, a member of the miR-16/15/195/424/497 family, has been demonstrated to act as a tumor suppressor in numerous types of malignant human tumors (7). Yang et al (8) found that miR-195 induced apoptosis in hepatocellular carcinoma cells in vitro through targeting LATS2 (8). Furthermore, Mao et al (9) showed that miR-195 suppressed osteosarcoma cell invasion and migration in vitro through downregulating FASN. Moreover, Ding et al (10) proposed that miR-195 may exert its tumor suppressive function by downregulating numerous nuclear factor (NF) $-\kappa B$ ) downstream effectors through directly targeting IKK $\alpha$ and TAB. In addition, miR-195 has been found to enhance the sensitivity of cancer cells to chemotherapy, with Yang et al (11) showing that upregulation of miR-195 increases the sensitivity of breast cancer cells to adriamycin through targeting Raf-1.

CARMA3, also known as CARD10, is a member of the CARMA family, as are the other two members CARMA1 (CARD11) and CARMA2 (CARD14). These three proteins share similar structural motifs, including CARD, coiled-coil, PDZ, SRC homology 3 and guanylate kinase-like domains (12). As a scaffold protein, CARMA3 has been demonstrated to have an important role in tumorigenesis (13). Recently, CARMA3 was found to be overexpressed in colorectal cancer and to contribute to colorectal cancer cell growth by promoting cell cycle progression through NF- $\mathrm{kB}$-mediated upregulation of cyclin D1 (14).

However, the specific roles and underlying molecular mechanisms of miR-195 and CARMA3 in colorectal cancer cells are yet to be elucidated. The present study determined the expression of miR-195 and CARMA3 in colorectal cancer tissues and their matched adjacent tissues. CARMA3 was identified to be a novel target of miR-195. Moreover, the regulatory roles of CARMA3 and miR-195 in cell proliferation, colony-formation and invasion were investigated in colorectal cancer cells in vitro. 


\section{Materials and methods}

Tissue specimen collection. The protocols used in the present study were approved by the Ethical Committee of Central South University (Changsha, China). Twenty colorectal cancer and matched adjacent tissues were obtained from the Department of General Surgery, the Third Xiangya Hospital of Central South University (Changsha, China) between September 2012 and March 2013. Informed consent was obtained from each patient included in this study. Each tissue sample was immediately frozen in liquid nitrogen following surgical removal.

Cell culture. SW480 and HT29 human colorectal cancer cell lines were obtained from the Cell Bank of Central South University (Changsha, China). Cells were cultured in Dulbecco's modified Eagle's medium (DMEM) supplemented with $10 \%$ fetal bovine serum (FBS), $100 \mathrm{IU} / \mathrm{ml}$ penicillin and $100 \mu \mathrm{g} / \mathrm{ml}$ streptomycin sulfate at $37^{\circ} \mathrm{C}$ in a humidified incubator containing $5 \% \mathrm{CO}_{2}$.

RNA extraction and quantitative polymerase chain reaction $(q P C R)$ analysis. Total RNA was extracted using TRIzol ${ }^{\circledR}$ reagent (Invitrogen Life Technologies, Carlsbad, CA, USA), according to the manufacturer's instructions. qPCR analysis was performed to assess mRNA expression using a TaqMan ${ }^{\circledR}$ Reverse Transcription kit and a Power SYBR ${ }^{\circledR}$ Green kit (Thermo Fisher Scientific, Waltham, MA, USA). Glyceraldehyde-3-phosphate dehydrogenase (GAPDH) was used as an endogenous control. For the analysis of miRNA expression, 10 ng RNA was converted to cDNA using the Applied Biosystems ${ }^{\circledR}$ TaqMan $^{\circledR}$ MicroRNA Reverse Transcription kit (Applied Biosystems, Carlsbad, CA, USA). qPCR was performed using the Applied Biosystems 7500 thermocycler (Applied Biosystems). U6 expression was used as an endogenous control. Relative mRNA and miRNA expression were analyzed using the $2^{-\Delta \Delta \mathrm{Ct}}$ method. The following primers were used in the qPCR analysis: Forward: 5'-CTGTGGGAGCGAATCGAGG-3' and reverse: 5'-CAG CGCAAGATGTCCATCA-3' for CARMA3; and forward: 5'-CTGGGCTACACTGAGCACC-3' and reverse: 5'-AAG TGGTCGTTGAGGGCAATG-3' for GAPDH. The primer sequences used to detect anti-matrix metalloproteinase (MMP)-9 were as follows: Forward: 5'-TGTACCGCTATG GTTACACTCG-3'; and reverse: 5'-GGCAGGGACAGTTGCT TCT-3'.

Western blot analysis. Tissues and cells were lysed in cold radioimmunoprecipitation assay lysis buffer. Proteins were separated using $10 \%$ SDS-PAGE and transferred onto polyvinylidene difluoride (PVDF) membranes (Invitrogen Life Technologies), which were then incubated with trisbuffered saline and Tween-20 containing 5\% skimmed milk at $4^{\circ} \mathrm{C}$ overnight. Membranes were then incubated with anti-CARMA3, and anti-GAPDH primary antibodies (Abcam PLC., Cambridge, UK), at room temperature for $3 \mathrm{~h}$. Subsequent to washing with phosphate-buffered saline and Tween-20 three times, PVDF membranes was incubated for $1 \mathrm{~h}$ with corresponding rabbit anti-mouse horseradish peroxidase-conjugated secondary antibodies (Abcam PLC.). Chemiluminescent detection was performed using an enhanced chemiluminescence kit (Pierce Chemical Co., Rockford, IL, USA). Image-Pro Plus 6.0 software (Media Cybernetics, Inc., Rockville, MD, USA) was used to analyze the relative target gene protein expression, which was normalized using GAPDH protein expression.

Transfection. Transfection was performed using Lipofectamine ${ }^{\circledR} 2000$ (Invitrogen Life Technologies) according to the manufacturer's instructions. For the functional analysis of miR-195, SW480 and HT29 cells were transfected with scrambled miRNA as a negative control (NC), miR-195 mimics, or an miR-195 inhibitor (Invitrogen Life Technologies). For the functional analysis of CARMA3, SW480 and HT29 cells were transfected with CARMA3-pcDNA3.1.

Dual Luciferase reporter assays. A Quick-Change ${ }^{\circledR}$ Site-Directed Mutagenesis kit (Stratagene California, La Jolla, CA, USA) was used to generate a mutant 3' UTR of CARMA3. The wild type 3' UTR of CARMA3 and the mutant 3' UTR of CARMA3 were then inserted into the psiCHECK $^{\mathrm{TM}}-2$ Vector (Promega Corporation, Madison, WI, USA). SW480 and HT29 cells were cultured to approximately 50-60\% confluence on 24-well plates, prior to co-transfection with psiCHECK $^{\mathrm{TM}}$-2-CARMA3-3' UTR or psiCHECK ${ }^{\mathrm{TM}}$-2-mutant CARMA3-3' UTR vector, with or without $50 \mathrm{nM}$ miR-195, using the Cellfectin ${ }^{\circledR}$ II Reagent (Invitrogen Life Technologies). The luciferase activity was assessed $48 \mathrm{~h}$ following transfection using a Beckman Coulter LD 400 luminometer (Beckman Coulter Inc., Fullerton, CA, USA). Renilla luciferase activity was normalized to firefly luciferase activity.

Cell proliferation assay. The MTT assay was used to measure cell proliferation. At $48 \mathrm{~h}$ post-transfection, the transfection medium in each well was replaced with $100 \mu \mathrm{l}$ fresh serum-free medium containing $0.5 \mathrm{~g} / \mathrm{l}$ MTT. Subsequent to incubation at $37^{\circ} \mathrm{C}$ for $4 \mathrm{~h}$, the MTT medium was removed by aspiration and $50 \mu 1$ dimethylsulfoxide was added to each well. Following incubation at $37^{\circ} \mathrm{C}$ for a further $10 \mathrm{~min}$, the optical density at $570 \mathrm{~nm}$ was measured using the Bio-Tek ${ }^{\mathrm{TM}}$ ELX-800 ${ }^{\mathrm{TM}}$ Absorbance Microplate reader (Biotek, Winooski, VT, USA). This experiment was repeated three times.

Cell invasion assay. A cell suspension containing $5 \times 10^{5}$ cells $/ \mathrm{ml}$ was prepared using serum-free DMEM. For the cell invasion assay, $500 \mu \mathrm{l}$ DMEM containing 10\% FBS was added to the upper chamber. Following incubation at $37^{\circ} \mathrm{C}$ with $5 \% \mathrm{CO}_{2}$ for $24 \mathrm{~h}$, the non-invading cells and the extracellular matrix gel were gently removed. Cells that had invaded through the membrane were stained for $20 \mathrm{~min}$, washed with water and then dried in air. Five fields were randomly selected and the number of cells which had invaded through the membrane were counted using a microscope (MVX10; Olympus, Tokyo, Japan).

Statistical analysis. All data are presented as the mean \pm standard deviation of at least three independent samples. Statistical analysis was performed using one-way analysis of variance or Student's t-tests using SPSS 19.0 (SPSS, Inc., IBM, Armonk, NY, USA) software. $\mathrm{P}<0.05$ was considered to indicate a statistically significant difference. 


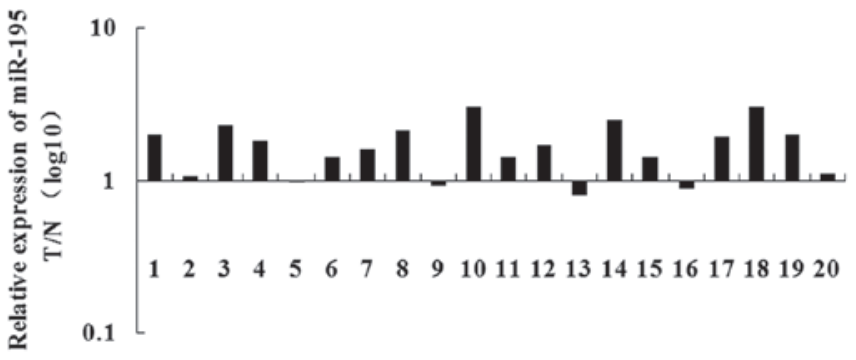

Figure 1. miR-195 is upregulated in colorectal cancer tissue. Quantitative polymerase chain reaction analysis was performed to determine the expression of miR-195 in tissue from 20 patients with colorectal cancer and the matched adjacent normal tissue. miR-195, microRNA-195; T/N, tumor/normal.

\section{Results}

miR-195 expression is downregulated in colorectal cancer tissues. miR-195 expression was determined in human colorectal cancer tissues and their matched adjacent tissues. As shown in Fig. 1, the expression of miR-195 was found to be increased in colorectal cancer tissues compared with their matched adjacent tissues. This finding suggests that miR-195 upregulation may have a role in colorectal cancer.

CARMA3 is a novel target of miR-195. Bioinformatic predication showed that the putative binding site for miR-195 at the 3' UTR of CARMA3 is highly conserved. In the present study, a mutant CARMA3 3' UTR was generated (Fig. 2A). As shown in Fig. 2B, luciferase activity was significantly reduced following co-transfection with the wild type CARMA3 3' UTR and miR-195 in SW480 and HT29 cells $(\mathrm{P}<0.01)$. However, the luciferase activity in other groups showed no significant differences. These findings suggest that CARMA3 is a novel target of miR-195 in SW480 and HT29 colorectal cancer cells.

CARMA3 expression is increased in colorectal cancer tissues. qPCR analysis was performed in order to determine the expression of CARMA3 in human colorectal cancer and matched adjacent normal tissues. As shown in Fig. 3, CARMA3 mRNA expression was observed to be downregulated in colorectal cancer tissues compared with the matched adjacent tissues. This suggests that CARMA3 may have a suppressive role in colorectal cancer.

miR-195 negatively regulates CARMA3 protein expression in SW480 and HT29 cells. The effect of miR-195 expression on CARMA3 protein expression in SW480 and HT29 cells was investigated. Subsequent to the transfection of SW480 and HT29 cells with an miR-195 mimic or inhibitor, the level of miR-195 expression was determined. As demonstrated in Fig. 4A, compared with the control group, miR-195 expression was significantly upregulated and downregulated following transfection with the miR-195 mimic and inhibitor, respectively $(\mathrm{P}<0.01)$. Therefore, the transfection efficiency was condsidered satisfactory. CARMA3 protein expression was then assessed using western blot analysis. CARMA3 protein expression was observed to be reduced in the cells overexpressing miR-195, but increased in the cells with
A
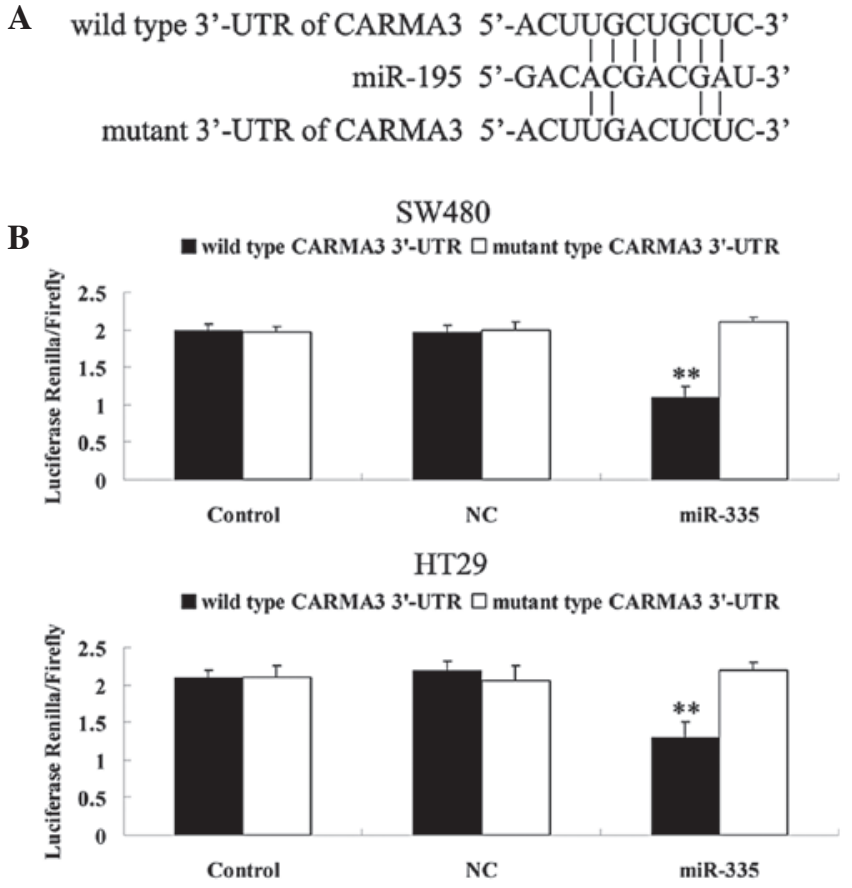

Figure 2. CARMA3 is a novel target of miR-195. (A) Seed region of miR-195 in the wild type 3'-UTR of CARMA3 revealed using bioinformatic analysis. A mutant CARMA3 3'-UTR was generated through the substitution of CUG to ACU within the seed region. (B) The luciferase reporter assay was used to determine whether CARMA3 was a target of miR-195. A wild type and mutant CARMA3 3'-UTR were subcloned into psiCHECK ${ }^{\mathrm{TM}}-2$ reporter vectors. PsiCHECK-2-CARMA3-3'-UTR or psiCHECK-2-mutant-CARMA3-3'-UTR vectors with $50 \mathrm{nM}$ miR-195 or scramble negative control miRNA were co-transfected into the SW480 and HT29 cells. ${ }^{* *} \mathrm{P}<0.01$ vs. control. Control, untransfected cells; NC, cells transfected with scramble negative control miRNA; mir-195, microRNA-195; UTR, untranslated region.

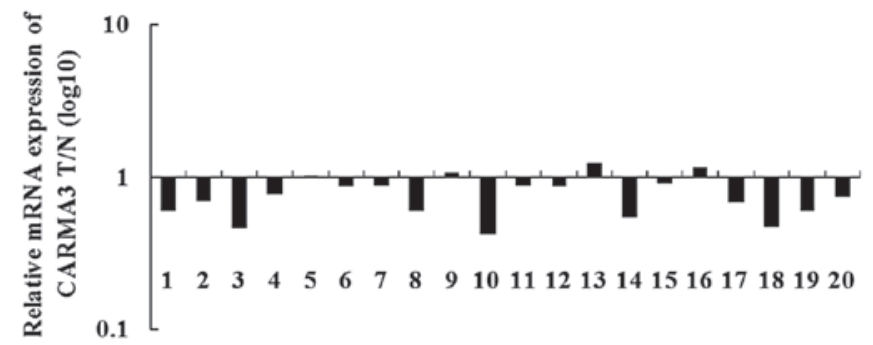

Figure 3. CARMA3 expression is reduced in colorectal cancer tissues. Quantitative polymerase chain reaction analysis was performed to determine the mRNA expression of CARMA3 in tissue from 20 patients with colorectal cancer and the matched adjacent normal tissue. miR-195, microRNA-195; $\mathrm{T} / \mathrm{N}$, tumor/normal.

downregulated miR-195 expression (Fig. 4B). These findings suggest that miR-195 negatively regulates the protein expression of CARMA3 in colorectal cancer cells.

miR-195 downregulates colorectal cancer cell proliferation through targeting CARMA3. To investigate the roles of CARMA3 and miR-195 in colorectal cancer cells, SW480 and HT29 cells were either transfected with an miR-195 mimic or inhibitor, or co-transfected with an miR-195 mimic and CARMA3-pcDNA3.1. As shown in Fig. 5A, transfection with CARMA3-pcDNA3.1 was found to reverse the inhibitory effect 


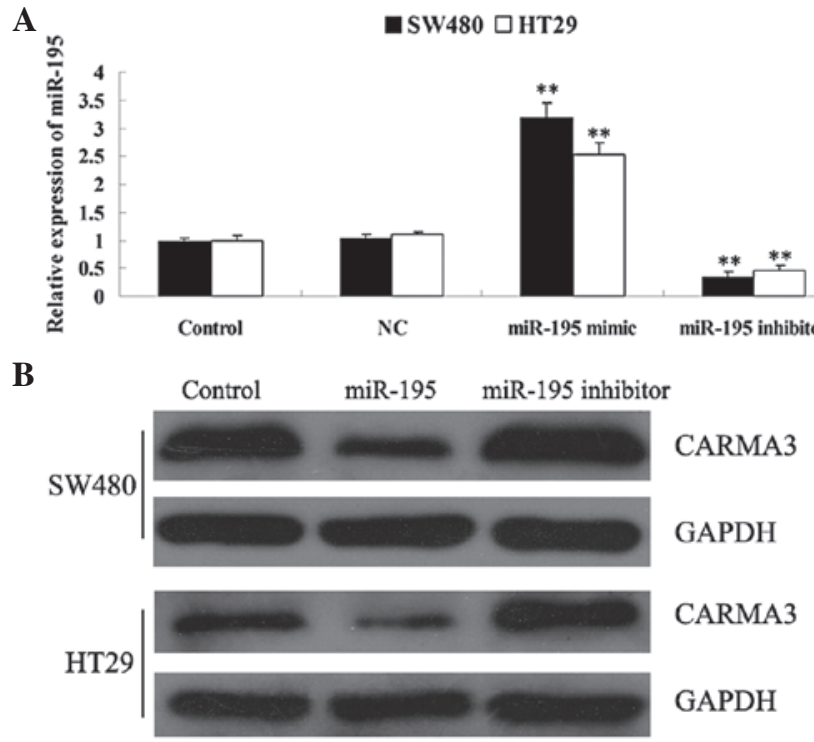

Figure 4. miR-195 negatively regulates CARMA3 protein expression in SW480 and HT29 cells. (A) Quantitative polymerase chain reaction was performed to assess miR-195 expression in SW480 and HT29 colorectal cancer cells transfected with scramble negative control miRNA, an miR-195 mimic or an miR-195 inhibitor. U6 expression was used as an endogenous control. ${ }^{* *} \mathrm{P}<0.01$ vs. control. (B) Western blot analysis was performed to determine the protein expression of CARMA3 in SW480 and HT29 cells transfected with scramble negative control miRNA, an miR-195 mimic or an miR-195 inhibitor. GAPDH expression was used as an endogenous control. Control, untransfected cells; $\mathrm{NC}$, cells transfected with scramble negative control miRNA; mir-195, microRNA-195.

of miR-195 on CARMA3 protein expression in SW480 and HT29 cells. Moreover, the cell proliferation assay revealed that miR-195-overexpression suppressed proliferation in colorectal cancer cells, but that downregulation of miR-195 promoted colorectal cancer cell proliferation. Furthermore, overexpression of CARMA3 was observed to reverse the inhibitory effect of miR-195 on SW480 and HT29 cell proliferation (Fig. 5B). These findings suggest that miR-195 may have an inhibitory role in colorectal cancer cell proliferation through directly inhibiting CARMA3 expression.

miR-195 suppresses colony formation in colorectal cancer cells through inhibiting CARMA3. The roles of miR-195 and CARMA3 in the regulation of colony formation in SW480 and HT29 colorectal cancer cells were investigated. As shown in Fig. 6, overexpression of miR-195 was found to reduce colony formation in SW480 and HT29 cells compared with the control group. However, downregulation of miR-195 was observed to promote colony formation in SW480 and HT29 cells. Moreover, the suppressive effect of miR-195 on colony formation in colorectal cancer cells was reversed with the overexpression of CARMA3. These findings suggest that miR-195 downregulates the malignant characteristics of colorectal cancer cells through inhibiting CARMA3.

miR-195 inhibits colorectal cancer cell invasion through targeting CARMA3. The roles of CARMA3 and miR-195 in the regulation of invasion in SW480 and HT29 cells were investigated. As shown in Fig. 7A, overexpression of miR-195 significantly inhibited SW480 and HT29 cell invasion, whereas
A

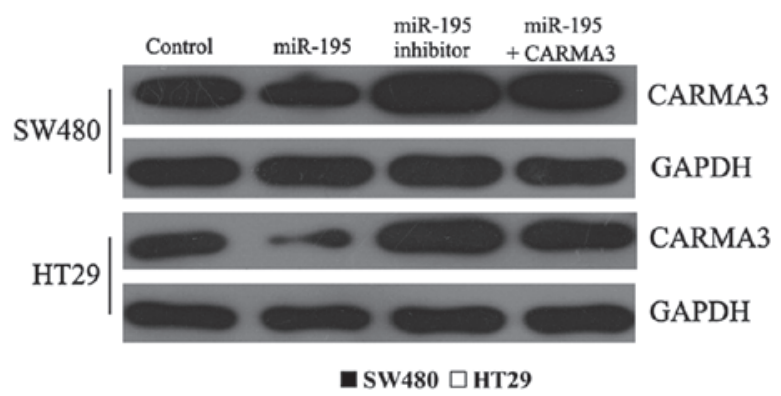

B

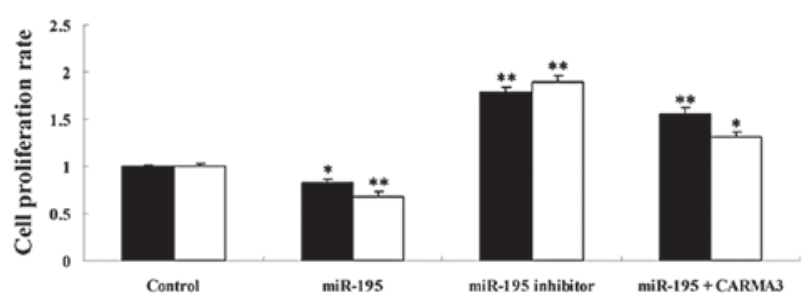

Figure 5. miR-195 downregulates SW480 and HT29 cell proliferation through downregulating CARMA3. (A) Western blot analysis was performed to determine CARMA3 protein expression in SW480 and HT29 cells transfected with an miR-195 mimic or an miR-195 inhibitor, or co-transfected with an miR-195 mimic and CARMA3-pcDNA3.1. GAPDH expression was used as an endogenous control. (B) Cell proliferation assay was performed to determine the effect of miR-195 and CARMA3 on SW480 and HT29 cell proliferation following transfection with an miR-195 mimic or miR-195 inhibitor, or co-transfected with an miR-195 mimic and CARMA3-pcDNA3.1. ${ }^{*} \mathrm{P}<0.05$ and ${ }^{* *} \mathrm{P}<0.01$ vs. control. Control, untransfected cells; mir-195, microRNA-195.

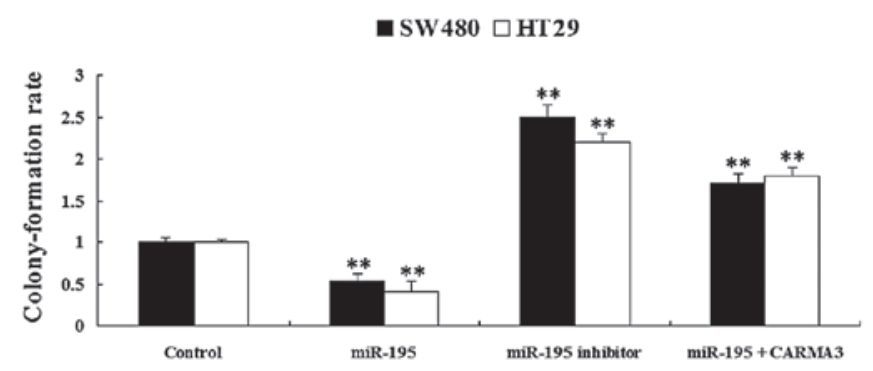

Figure 6. miR-195 downregulates colony-formation in SW480 and HT29 cells through suppressing CARMA3. Colony formation assays were performed to determine the effect of miR-195 and CARMA3 on colony formation in SW480 and HT29 cells transfected with an miR-195 mimic or an miR-195 inhibitor, or co-transfected with an miR-195 mimic and CARMA3-pcDNA3.1. ** P $<0.01$ vs. control. Control, untransfected cells; mir-195, microRNA-195.

downregulation of miR-195 significantly promoted SW480 and HT29 cell invasion. Moreover, the inhibitory effect of miR-195 on cell invasion was reversed with the overexpression of CARMA3, suggesting that miR-195 has an inhibitory role in the regulation of colorectal cancer cell invasion through downregulating CARMA3.

Recently, Feng et al (15) reported that CARMA3 promoted glioma cell invasion through MMP-9, a key tumor invasion factor. Thus, to investigate the mechanism by which miR-195 regulates colorectal cancer cell invasion, MMP-9 mRNA expression was assessed in each group. As shown in Fig. 7B, MMP-9 expression was significantly reduced in the SW480 and HT29 cells overexpressing miR-195, while significantly increased in the cells with downregulated miR-195 expression. 
A

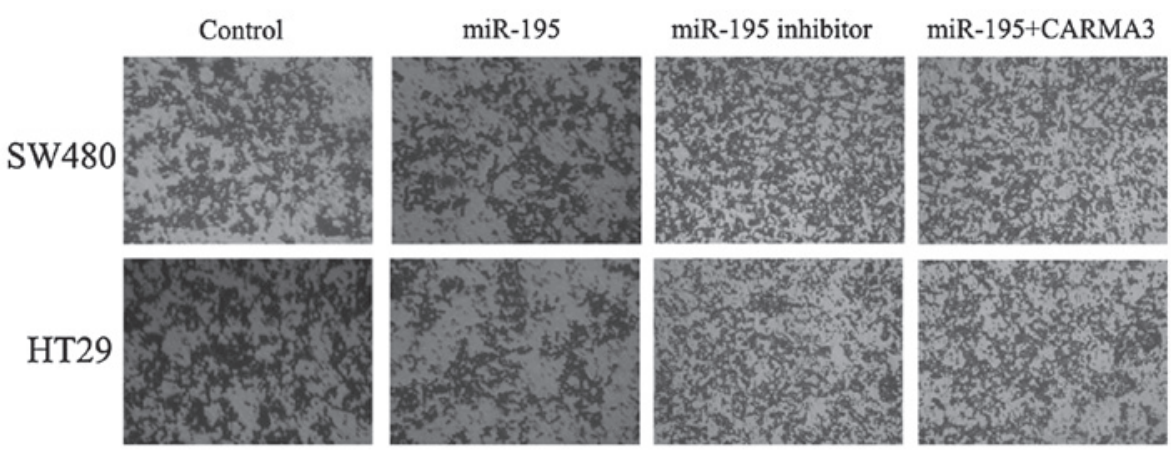

$\square$ SW480 $\square$ HT29

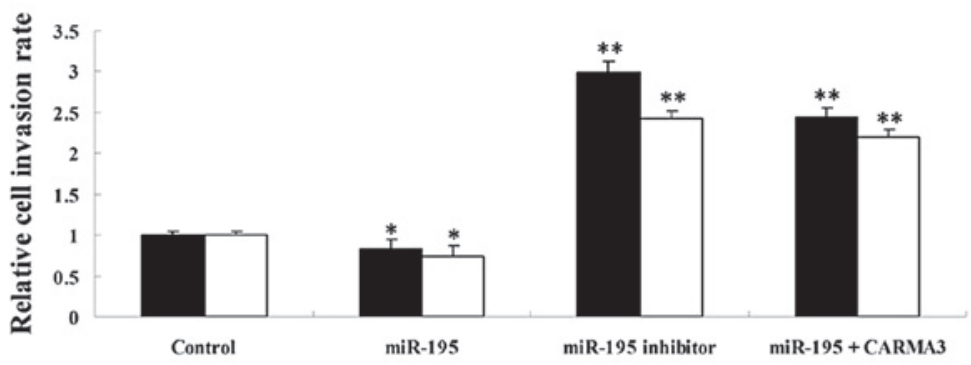

SW480 $\square$ HT29

$\mathbf{B}$

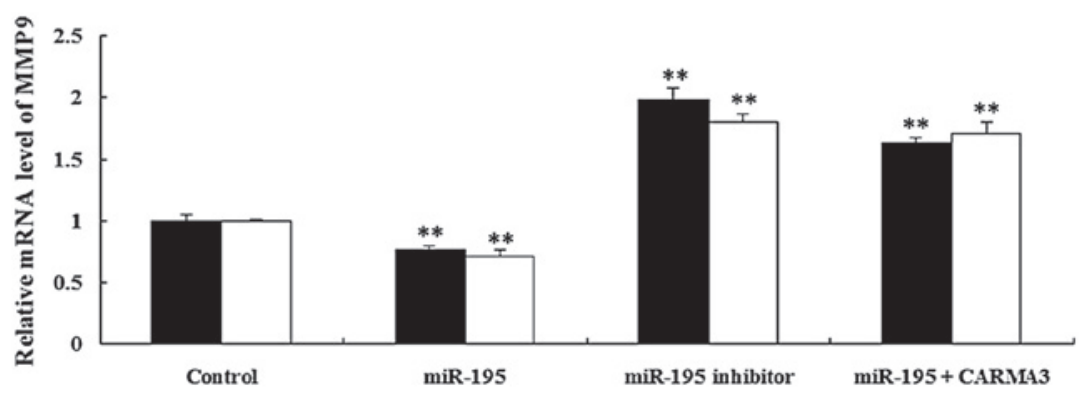

Figure 7. miR-195 inhibits invasion in SW480 and HT29 cells through modulating MMP-9 by targeting CARMA3. (A) Cell invasion assay was performed to determine the effect of miR-195 and CARMA3 on invasion in SW480 and HT29 cells following transfection with an miR-195 mimic or an miR-195 inhibitor, or co-transfected with an miR-195 mimic and CARMA3-pcDNA3.1. ${ }^{~} \mathrm{P}<0.05$ and ${ }^{* *} \mathrm{P}<0.01$ vs. control. (B) Quantitative polymerase chain reaction was performed to assess MMP-9 mRNA expression in SW480 and HT29 cells transfected with an miR-195 mimic or an miR-195 inhibitor, or co-transfected with an miR-195 mimic and CARMA3-pcDNA3.1. ${ }^{* *} \mathrm{P}<0.01$ vs. control. Control, untransfected cells; mir-195, microRNA-195; MMP, matrix metalloproteinase.

Furthermore, CARMA3 overexpression was found to reverse the suppressive effect of miR-195 on MMP-9 expression in SW480 and HT29 cells. These findings suggest that the effect of miR-195 on colorectal cancer cell invasion may involve the modulation of MMP-9 through targeting CARMA3.

\section{Discussion}

It is well established that miRNAs have a crucial role in the regulation of various human malignancies, due to their capacity to regulate the expression of numerous target genes, which are associated with tumorigenesis, tumor progression, metastasis and sensitivity to chemotherapy (16-18). The development and progression of colorectal cancer has been shown to be mediated by complex molecular events, which monitor cell proliferation and motility (19). In the present study, miR-195 downregulation was found to correlate with CARMA3 upregulation in colorectal cancer tissues. Furthermore, CARMA3 was identified to be a novel target of miR-195, with miR-195 negatively regulating the protein expression of CARMA3 in colorectal cancer cells. Functional analyses demonstrated that miR-195 had an inhibitory effect on cell proliferation, colony formation and invasion in colorectal cancer cells, at least in part through targeting CARMA3.

It has been demonstrated that miR-195 is involved in colorectal cancer (20-23). Wang et al (21) showed that miR-195 was downregulated in colorectal cancer tissues, which is consistent with the findings of the present study. Moreover, Wang et al (21) showed that the downregulation of miR-195 in colorectal cancer was correlated with lymph node metastasis and poor prognosis, indicating that miR-195 may have the potential to be an independent marker for the predication of clinical outcome in patients with colorectal cancer. In addition, miR-195 has been found to promote apoptosis and suppress tumorigenicity in human colorectal cancer cells through targeting Bcl-2, an important anti-apoptotic gene (22). Furthermore, miR-195 has been reported to enhance the susceptibility of colorectal cancer cells to the chemotherapeutic drug doxorubicin through directly inhibiting the expression of the BCL2L2(23). These findings suggest that miR-195 has an 
inhibitory role in colorectal cancer and may be a promising therapeutic candidate for colorectal cancer.

A single miRNA is capable of regulating numerous target genes; therefore, in the present study it was hypothesized that other genes may also be involved in miR-195-mediated biological processes in colorectal cancer cells. In order to investigate this hypothesis, bioinformatic analyses were performed and CARMA3 was identified to be a target of miR-195. A luciferase reporter assay further confirmed that CARMA3 was a target of miR-195 in colorectal cancer cells. CARMA3 has recently been shown to have an oncogenic role in the progression of several human malignancies $(12-14,24,25)$. Wu et al (24) showed that CARMA3 was upregulated in renal cell carcinoma and that its overexpression may be an independent prognostic indicator of survival in patients with renal cell carcinoma. Furthermore, Li et al (12) reported that overexpression of CARMA3 in non-small-cell lung cancer is associated with tumor progression. Recently, Miao et al (14) reported that CARMA3 was overexpressed in colorectal cancer, consistent with the findings of the present study. Miao et al (14) also found that the overexpression of CARMA3 in colorectal cancer was associated with tumor-node-metastasis stage, lymph node metastasis and Ki67 proliferation index, indicating that CARMA3 may act as an oncogene in colorectal cancer. CARMA3 was also found to enhance colorectal cancer cell proliferation in vitro, by promoting cell cycle progression through $\mathrm{NF}-\kappa \mathrm{B}$-induced upregulation of cyclin D1 (14). However, the role of CARMA3 and miRNAs in the regulation of colorectal cancer is yet to be elucidated. Therefore, in the present study, the interelation between miR-195 and CARMA3 was investigated in colorectal cancer cells. miR-195 overexpression was found to significantly downregulate CARMA3 protein expression, while inhibition of miR-195 was observed to significantly promote CARMA3 protein expression in colorectal cancer cells. These findings suggest that miR-195 negatively regulates CARMA3. Moreover, these findings suggest that miR-195 inhibited colorectal cancer cell proliferation, colony-formation and invasion through directly suppressing CARMA3 expression.

CARMA3 has previously been reported to be required for $\mathrm{G}$ protein-coupled receptor induced $\mathrm{NF}-\kappa \mathrm{B}$ activation, which participates in the regulation of MMP-9, a key promoter of cancer cell invasion $(26,27)$. Therefore, in the present study the expression of MMP-9 was assessed. MMP-9 mRNA expression was found to correlate with colorectal cancer cell invasion, indicating that the inhibitory effect of miR-195 on colorectal cancer cell invasion may at least partially be mediated through the downregulation of MMP-9, by directly targeting CARMA3. In accordance with these findings, Feng et al (15) reported that CARMA3 promoted glioma cell invasion through upregulating the expression of MMP-9.

In conclusion, the present study demonstrates that miR-195 inhibits colorectal cancer cell proliferation, colony formation and invasion, at least partially through targeting CARMA3. These findings suggest that miR-195 may be a potential candidate for the treatment of colorectal cancer.

\section{References}

1. Boncheva V, Bonney SA, Brooks SE, et al: New targets for the immunotherapy of colon cancer-does reactive disease hold the answer? Cancer Gene Ther 20: 157-168, 2013.
2. Zhang $\mathrm{N}, \mathrm{Li} \mathrm{X}, \mathrm{Wu} \mathrm{CW}$, et al: microRNA-7 is a novel inhibitor of YY1 contributing to colorectal tumorigenesis. Oncogene 32: 5078-5088, 2012.

3. Yates LA, Norbury CJ and Gilbert RJ: The long and short of microRNA. Cell 153: 516-519, 2013.

4. Dassow H and Aigner A: MicroRNAs (miRNAs) in colorectal cancer: from aberrant expression towards therapy. Curr Pharm Des 19: 1242-1252, 2013.

5. Dong Y, Zhao J, Wu CW, et al: MiR-133a activates the p53/p21 pathway and functions as a tumor suppressor in colorectal cancer by repressing RFFL. Mol Cancer Res 11: 1051-1060, 2013.

6. Yu Y, Sarkar FH and Majumdar AP: Down-regulation of miR-21 induces differentiation of chemoresistant colon cancer cells and enhances susceptibility to therapeutic regimens. Transl Oncol 6: 180-186, 2013.

7. He JF, Luo YM, Wan XH and Jiang D: Biogenesis of MiRNA-195 and its role in biogenesis, the cell cycle, and apoptosis. J Biochem Mol Toxicol 25: 404-408, 2011.

8. Yang X, Yu J, Yin J, Xiang Q, Tang H and Lei X: MiR-195 regulates cell apoptosis of human hepatocellular carcinoma cells by targeting LATS2. Pharmazie 67: 645-651, 2012.

9. Mao JH, Zhou RP, Peng AF, et al: microRNA-195 suppresses osteosarcoma cell invasion and migration in vitro by targeting FASN. Oncol Lett 4: 1125-1129, 2012.

10. Ding J, Huang S, Wang Y, et al:: Genome-wide screening reveals that miR-195 targets the TNF- $\alpha / \mathrm{NF}-\kappa \mathrm{B}$ pathway by down-regulating IкB kinase alpha and TAB3 in hepatocellular carcinoma. Hepatology 58: 654-666, 2013.

11. Yang G, Wu D, Zhu J, et al: Upregulation of miR-195 increases the sensitivity of breast cancer cells to Adriamycin treatment through inhibition of Raf-1. Oncol Rep 30: 877-889, 2013.

12. Li Z, Qu L, Dong Q, et al: Overexpression of CARMA3 in non-small-cell lung cancer is linked for tumor progression. PLoS One 7: e36903, 2012.

13. Zhao T, Miao Z, Wang Z, et al: CARMA3 overexpression accelerates cell proliferation and inhibits paclitaxel-induced apoptosis through NF- $\kappa$ B regulation in breast cancer cells. Tumour Biol 34: 3041-3047, 2013

14. Miao Z, Zhao T, Wang Z, et al: CARMA3 is overexpressed in colon cancer and regulates NF- $\kappa B$ activity and cyclin D1 expression. Biochem Biophys Res Commun 425: 781-787, 2012.

15. Feng X, Miao G, Han Y and Xu Y: CARMA3 is overexpressed in human glioma and promotes cell invasion through MMP9 regulation in A172 cell line. Tumour Biol: July 27, 2013 (Epub ahead of print).

16. Callegari E, Elamin BK, Sabbioni S, Gramantieri L and Negrini M: Role of microRNAs in hepatocellular carcinoma: a clinical perspective. Onco Targets Ther 6: 1167-1178, 2013.

17. Chou J, Shahi P and Werb Z: microRNA-mediated regulation of the tumor microenvironment. Cell Cycle 12: 3262-3271, 2013.

18. Bienertova-Vasku J, Sana J and Slaby O: The role of microRNAs in mitochondria in cancer. Cancer Lett 336: 1-7, 2013.

19. Hammoud SS, Cairns BR and Jones DA: Epigenetic regulation of colon cancer and intestinal stem cells. Curr Opin Cell Biol 25: 177-183, 2013.

20. Piepoli A, Tavano F, Copetti M, et al: Mirna expression profiles identify drivers in colorectal and pancreatic cancers. PLoS One 7: e33663, 2012.

21. Wang X, Wang J, Ma H, Zhang J and Zhou X: Downregulation of miR-195 correlates with lymph node metastasis and poor prognosis in colorectal cancer. Med Oncol 29: 919-927, 2012.

22. Liu L, Chen L, Xu Y, Li R and Du X: microRNA-195 promotes apoptosis and suppresses tumorigenicity of human colorectal cancer cells. Biochem Biophys Res Commun 400: 236-240, 2010.

23. Qu J, Zhao L, Zhang P, et al: MicroRNA-195 chemosensitizes colon cancer cells to the chemotherapeutic drug doxorubicin by targeting the first binding site of BCL2L2 mRNA. J Cell Physiol: March 22, 2013 (Epub ahead of print).

24. Wu GL, Yuan JL, Huang XD, et al: Evaluating the expression of CARMA3 as a prognostic tumor marker in renal cell carcinoma. Tumour Biol 34: 3431-3435, 2013.

25. Rehman AO and Wang CY: CXCL12/SDF-1 alpha activates NF-kappaB and promotes oral cancer invasion through the Carma3/Bcl10/Malt1 complex. Int J Oral Sci 1: 105-118, 2009.

26. Sun J: CARMA3: A novel scaffold protein in regulation of NF- $\kappa$ B activation and diseases. World J Biol Chem 1: 353-361, 2010.

27. Dilly AK, Ekambaram P, Guo Y, et al: Platelet-type 12-lipoxygenase induces MMP9 expression and cellular invasion via activation of PI3K/Akt/NF-אB. Int J Cancer 133: 1784-1791, 2013. 